\title{
The Design of Two-Wheeled Robotic Self-Balancing Walking Control System
}

\author{
Lingling Zhong ${ }^{1+}, \quad$ Teng $\mathrm{Lv}^{2}$, and Kang Liu ${ }^{1}$ \\ ${ }^{1}$ School of Electronic and Communication Engineering, Anhui Xinhua University, Hefei 230088, China \\ ${ }^{2}$ School of Information Engineering, Anhui Xinhua University, Hefei 230088, China
}

\begin{abstract}
Research on mobile robots has become a popular field in robot research because of the broad application prospects of mobile robots in all walks of life. Considering the complicated designs and high prices of the Humanoid robots' different DoF (Degree of Freedom), a two-wheeled robotic self-balancing walking control system is designed in the paper. The system uses the microcontroller as the control core, the attitude sensor to collect the angular velocity and acceleration data, and the Kalman filter to perform data fusion to obtain the optimal inclination and to realize the tilt control. It also uses the encoder to measure the walking speed to realize the speed control in the front and rear direction. The steering control of the robot is realized by the rotational difference between the two motors. The experiment proves that the two-wheeled robot can walk upright and keep balance, and has the advantages of simple structure, convenient parameter adjustment and low cost.
\end{abstract}

Keywords: Two-wheeled robot, tilt control, speed control, steering control.

\section{Introduction}

Today, mobile robots are widely used, and have begun to be applied in exploration, rescue, mining, entertainment and other fields. Among them, wheeled robots have become an area of researches on mobile robots because of their flexibility, simple structure, easy operation and implementation[1-2]. The twowheeled robot has a coaxial two-wheel structure, and it moves in parallel to the left and right. Its system is similar to the inverted pendulum system. Considering it demands strong stability in the work, the system proposes three PID (Proportion Integration Differentiation) control modes, which will superimpose and fuse the tilt control loop, speed control loop and steering control, calculate and output PWM (Pulse-Width Modulation) signals. Through them, the motion of the system motor is controlled. And the robot is operated upright and balanced, which has a strong anti-interference standing ability.

\section{Balance Control Principle}

The two-wheel self-balancing walking robot superimposes the three parameters of inclination, velocity and direction by collecting sensor data, generates control voltage, controls the movement of two motors to realize walking upright and balanced, and under the interference of external force, the robot remains standing upright by this intelligent system . The block diagram of the system's balance control is shown in Figure 1 . The first part is the tilt ring control, the second part is the speed loop control, and the third part is the direction control.

\footnotetext{
${ }^{+}$Corresponding author. Tel.: +861-370-5511342; fax: +86-551-65872323

E-mail address: zhll3610@163.com
} 


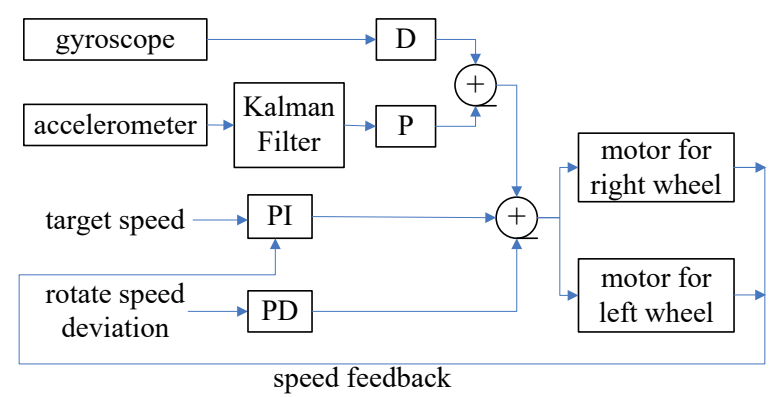

Fig. 1: The block diagram of the system's balance control.

Tilt control loop: it belongs to PD closed loop control. When the tilt occurs during the walking of the robot, the two motors are controlled to rotate the forward-reverse directions to control the rotation of the wheel, so the robot can keep upright, that is, when robot tilted to the right, the wheel accelerates to the right to form a restoring force to the left; When robot tilted to the left, the wheel accelerates to the left to form a restoring force to the right, which is a proportional $\mathrm{P}$ process. The tilt angle velocity and the tilt angle are collected by the sensor, and the Kalman filter optimal estimation method is used to fuse the two parameters to eliminate the drift caused by the system noise, so it can output the dynamic optimal angle value, and then calculate the angle to obtain the restoring force. The system upright process is like the inverted pendulum movement, it does not immediately return to the original vertical state once tilted, and needs to swing until it is balanced[3-5], so damping force is needed to speed up the system to maintain the upright balance, which is a differential D process. The tilt angular velocity is acquired by the sensor, and the angular velocity differential operation is performed to obtain the damping force.

Speed control loop: It belongs to PI closed loop control. The running speed is adjusted by the tilt angle of the robot instead of directly changing the rotational speed of the motor. If the motor speed is directly controlled, the robot in the balance will be dumped, which will seriously affect the stability of the system. The encoder is used to collect the current running speed of the robot and compare it with the expected target speed, generate the error speed, and then calculate the tilt angle proportionally to get the tilted angle, and then obtain the moving speed of the model by integral calculation. The integral I operation coefficient is the definition of the ability of the speed loop to suppress speed error. The larger the integral I operation coefficient, the stronger the ability to suppress the speed error, in the contrary, if the speed loop is controlled only by the integral operation, it is easy to generate integral error, and the robot is prone to oscillation during the running process[6]. Therefore, the proportional $\mathrm{P}$ operation is introduced to eliminate the oscillation phenomenon. Since the robot is a system that does not satisfy the minimum phase condition, in the speed control, the proportional operation coefficient and the adjusted speed should not be too large, otherwise it is easy to cause the system to generate positive feedback so that the robot may run out of control, so the speed needs to be slowly changed during the adjustment.

Steering control: It belongs to PD open loop control. The steering of the robot is controlled by the difference between the rotational speeds of the two motors, which is a simple proportional $\mathrm{P}$ operation. During the steering process of the robot, due to its own weight, there is a large moment of inertia, and the heavier its own weight, the larger the inertia value, so the differential D operation is introduced to eliminate the steering overshoot and avoid the robot deviating from its own travel line.

\section{System Hardware Design}

The two-wheeled robot control system is mainly composed of a microcontroller, two motors, a motor drive module, an attitude sensor, a wireless communication module, a liquid crystal display and a power supply module. The hardware block diagram of the system is shown in Figure 2. The microcontroller STM32f103t8u6 is a 32-bit ARM core-based microcontroller with an operating frequency of $72 \mathrm{MHz}$, with 64 or $128 \mathrm{k}$ bytes of flash memory, 20K bytes of SRAM, 7 timers, and 9 communication interfaces[7]. The STM32f103t8u6 consumes the lowest power of the 32-bit products among the market and consumes $0.5 \mathrm{~mA} / \mathrm{MHz}$. The LCD1602 displays data in real time for easy debugging and observation of data. The function of the key module is to set control parameters. 


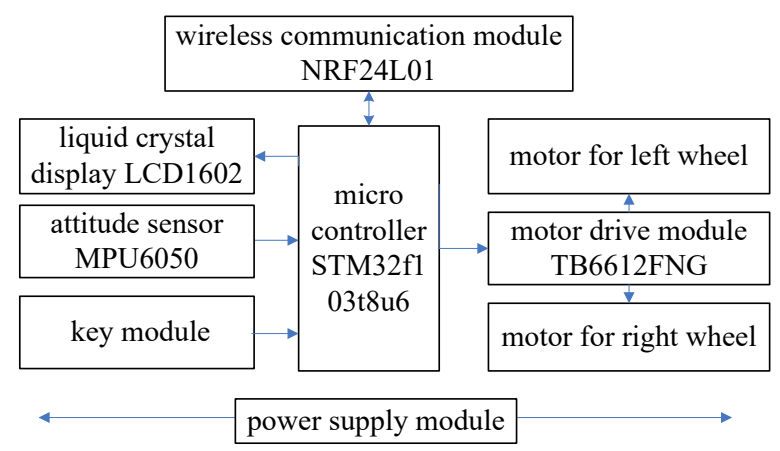

Fig. 2: The hardware block diagram of the system.

Attitude sensor: The MPU6050 is a six-axis sensor manufactured by InvenSense, which features light weight, low power loss, superior performance and low price. I2C communication is used to output the acceleration (accelerometer) and angular velocity (gyroscope) of the current system $\mathrm{x}$ and $\mathrm{y}$ axes in real time[8-9]. The PB8 and PB9 pins of the microcontroller are connected to the SDA and SCL pins of I2C respectively, and the acquired acceleration and angular velocity are combined by the Kalman filter optimal estimation method to output the optimal tilted angle of the robot.

Motor driver module: TB6612FNG is a new drive device that can independently control two motors bidirectionally. The peak current reaches $3.2 \mathrm{~A}$ and the maximum output current is $2 \mathrm{~A}$. It is a SMD chip with high integration[10-11]. The PB14, PB15, PB13 and PB12 of the microcontroller are connected to the input pins AIN1, AIN2, BIN1 and BIN2 respectively, and the drive motor is controlled to realize the forward and reverse rotation of the motor; PA3 and PA4 are respectively connected to the input pins PWMA and PWMB to control the driving motor and then change the rotational speed of the motor. Motor drive module output pins $\mathrm{AO} 1$ and $\mathrm{AO} 2, \mathrm{BO} 1$ and $\mathrm{BO} 2$ are connected to the left and right motor pins respectively to drive the motor to rotate. The PB7, PB6, PA7 and PA6 of the microcontroller are connected to the pins of the encoders in the left and right motors respectively to achieve the measurement of the running speed of the two wheels.

Wireless communication module: NRF24L01 is a wireless communication chip produced by NORDIC Company. It has low price and stable signal transmission. The purpose is to receive external information and realize the control of the robot.

\section{System Software Design}

The software design of the two-wheeled robot self-balancing walking control system uses $\mathrm{C}$ language to write the source code, which is compiled by Keil program[12]. The main functions of the system program are:

- The overall program flow of the control system: start, initialization, main function and end.

- Input and output operation interface of the system: parameter setting by using the button, data and status of the display screen.

- Calculation of the parameters of the system: calculating the tilted angle, the speed and the difference between the two wheels.

- PID control of the system: inclination control, speed control and direction control.

- Communication of the system: NRF data transmission and reception.

- Control of motor rotational speed: PWM output.

The program flow is shown in Figure 3. First, the initialization of each functional circuit, including STM32f103t8u6 initialization, MPU6050 initialization, LCD1602 initialization, key scan initialization, and NRF24L01 initialization; Second, various Flash parameters are initialized; Third, the main function operation, the program immediately starts to key scan, display refresh, NRF data transceiving, and receive data decoding according to timing. Since the three PID controls (inclination control, speed control, and direction control) require precise execution cycles, they are put into interrupts for execution, the main program and interrupt routine are used for data communication with the global variables; Finally, PWM outputs and controls the motor rotation. 


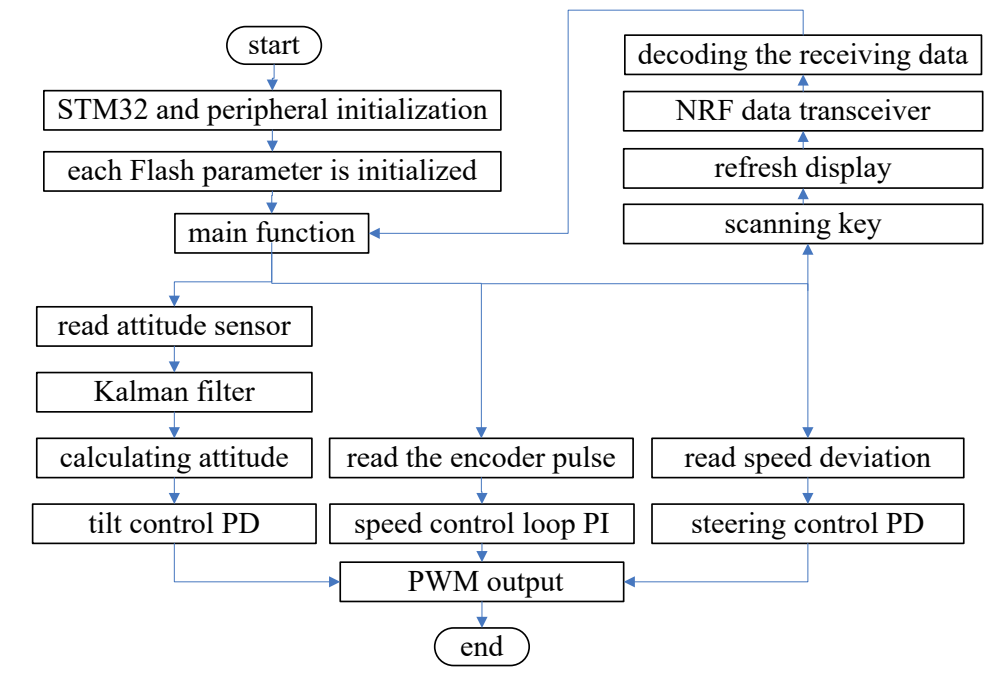

Fig. 3: The program flow chart.

\section{System Testing}

The PWM motor drive voltage generated by the microcontroller through the PID control algorithm is unipolar, frequency is $5 \mathrm{KHz}$, and the value range is $-1.0 \mathrm{~V} \sim 1.0 \mathrm{~V}[13]$. Robot's body part is shown in figure 4 . Its running speed to the front and tilted direction to the left are defined as positive value. The control coefficient is adjusted in the order of adjusting the proportional coefficient $\mathrm{P}$, then the differential coefficient $\mathrm{D}$, and finally the integral coefficient I. The target speed of the robot is 0 , that is, it is in the quiescent state. System test data is shown in Table 1.

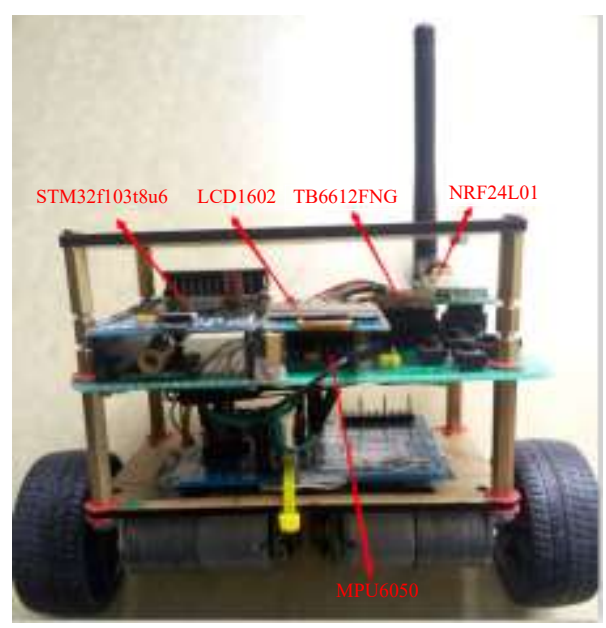

Fig. 4: Robot's body part.

Table 1: The system test data tables

\begin{tabular}{|c|c|c|c|}
\hline $\mathrm{P}$ & $\mathrm{I}$ & $\mathrm{D}$ & Motion Phenomena \\
\hline 0 & 0 & 0 & Dump under gravity \\
\hline 0.15 & 0 & 0 & $\begin{array}{c}\text { Stay upright for a short time, shake, and walk } \\
\text { forward }\end{array}$ \\
\hline 0.3 & 0 & 0 & Keep upright, shake, and walk forward \\
\hline 0.3 & 0 & 0.0015 & Keep upright, weakened jitter, and walk forward \\
\hline 0.3 & 0 & 0.004 & Keep upright, no jitter, and walk forward \\
\hline 0.3 & 0.015 & 0.004 & $\begin{array}{c}\text { Keep upright, no jitter, and walk back and forth } \\
\text { around a certain location }\end{array}$ \\
\hline 0.3 & 0.02 & 0.004 & Keep upright, no jitter, and stand still \\
\hline
\end{tabular}

It can be seen from the test data that the adjustment of the proportionality factor $\mathrm{P}$ can overcome the effect of the earth's gravity on the robot. As the $\mathrm{P}$ value increases, the robot gradually stands upright, but if the value is too large, the robot will start to vibrate and affect the upright balance. The differential coefficient 
$\mathrm{D}$ is introduced to eliminate the vibration phenomenon of the robot. The adjustment of the differential coefficient D counteracts the free energy brought by the free swing, overcomes the vibration of the system, and can restore the balance of the system under the interference of external forces. During the test, the robot has been walking to the right because the robot has an initial tilted angle, which is caused by insufficient accuracy of the hardware or measurement errors of the sensor. Introduce the integral coefficient I to eliminate the installation error of the system and make the robot stand still. The velocity integral error can prevent the robot from running in one direction, and as the value of I increases, the stopping speed of the robot is accelerated. When $\mathrm{P}=0.3, \mathrm{I}=0.02, \mathrm{D}=0.004$, the two-wheeled robot self-balancing walking control system has the best vertical balance and can be used as the operating parameters of the system.

\section{Conclusion}

In this paper, a two-wheeled robotic self-balancing walking control system is designed. The STM32f103t8u6 is used as the control core. The optimal tilted angle value is output by the Kalman filter algorithm to improve the accuracy of the tilted angle of the system. The PID algorithm is used to control the rotation of the two-wheeled motor. System tests have shown that the upright balanced walking function of the two-wheeled robot is realized.

\section{Acknowledgements}

The work is supported by Talent of Discipline and Specialty in Colleges and Universities of Anhui Province (No.gxbjZD54), Academic Leader Foundation (No.2014XXK06), and Introduction of Talents Foundation of Anhui Xinhua University (No.2015kyqd002), Reform Comprehensive Experimental Unit of Electronic Information Engineering Major (No.2015zy073).

\section{References}

[1] Qiyuan Fan, Xianghong Qin, Hongyi Li. Design of control system for two-wheel balancing robot based on STM32. Automation \& Instrumentation, 2018, 33(3):18-21.

[2] Guanyang Guan, Guangfeng Chen, Wei Xi. Research on two-wheel robot control based on Codesys. Automation \& Instrumentation, 2018, 33(4):16-19.

[3] Ebrahimi S, Mardany A. Dynamic modeling and construction of a two-wheeled mobile manipulator, part I: Selfbalancing. Rsi International Conference on Robotics \& Mechatronics. 2016.

[4] Chhotray A, Pradhan M K , Pandey K K , et al. Kinematic Analysis of a Two-Wheeled Self-Balancing Mobile Robot.Proceedings of the International Conference on Signal, Networks, Computing, and Systems. Springer India, 2016.

[5] Lingyan $\mathrm{Hu}$, Yuanchun $\mathrm{Xu}$, Shaoping Xu, et al. Two-wheel inspection robot based on kalman filter and linear quadratic adjustment. Computer Engineering, 2016, 42(1):304-310.

[6] Yingli Shu, Lei Kou, Quande Yuan, et al. Research on balancing movement strategy of two-wheeled robot based on double ultrasonic data fusion. Automation Instrumentation, 2017, 38(2):36-40.

[7] Jun, W., Zhimin, L., Fangguo, W.: The design and implementation of the two-wheeled balancing mobile robots based on STM32. Electron. World 7. 2016, pp.145-147 .

[8] MPU6050[EB/OL]. [2014-03-25]. https://www.cnblogs.com/firege/p/5806073.html.

[9] Genzai Fang, Fuhai Li. Design and implementation of attitude estimation system for small aircraft. Journal of Electronic Measurement and Instrumentation, 2017, 31(3):474-480.

[10] Ying Bai, Zvi S. Roth . Classical Linear Control Systems-PID Control Systems. Classical and Modern Controls with Microcontrollers. 2018, pp.195-201.

[11] Yinping Liu. MiroSot soccer robot motor drive system design. Automation Instrumentation, 2018, 39(3): 2-54.

[12] Tao Wu, Ru Bai, Zhu Liyao, et al. Design of attitude reference system based on kalman filter. Journal of Sensing Technology, 2016, 29(4):531-535.

[13] Yuan Xu, Weiting Liu, Haifeng Wei. Two-wheel self-balancing robot control under multi-sensor filtering algorithm. Information Technology, 2018, (4): 27-32. 